International Journal of Biomedicine | June 2021 - Volume 11, Issue Suppl_1: Abstracts from the Third Russian International Conference "Cryo-electron microscopy 2021: achievements and prospects"

\author{
POSTER ABSTRACT PRESENTATIONS
}

SESSION TITLE: EM RESEARCH RELATED TO MEDICINE

DOI: 10.21103/JJBM.11.Suppl_1.P36

\title{
Abstract P-36: Structural Analysis of Conformational Changes of Bacterial and Eukaryotic Tubulins
}

\author{
$\underline{\text { Liubov O. Makarova }}^{1,2}$, Alena V. Korshunova ${ }^{3}$ \\ ${ }^{I}$ Moscow Institute of Physics and Technology, Dolgoprudny, Russia \\ ${ }^{2}$ Physics Department, Lomonosov Moscow State University, Moscow, Russia \\ ${ }^{3}$ Center for Theoretical Problems of Physicochemical Pharmacology, Russian \\ Academy of Sciences, Moscow, Russia
}

Background: Eukaryotic $\alpha$ - and $\beta$-tubulin proteins stand out among tubulin-like proteins by their ability to form hollow dynamically unstable microtubules (MT) with 13 protofilaments. Microtubules are part of the cell cytoskeleton and play a key role in chromosome division in mitosis. A considerable amount of anticancer drugs works on microtubules level breaking its dynamic. But the mechanism of dynamic instability and works of these drugs remains unknown. Bacteria of the genus Prostecobacter have unique bacterial tubulins (BtubA/B) capable to form hollow dynamically unstable 5 protofilament MTs (miniMT). Instead of great differences, both tubulins have many common features. Eukaryotic tubulin was known to have structural changes through GTP hydrolysis (compactization for approximately $2 \AA$ and a twist for $0,1^{\circ}$ ). «Anchor point» structure in alpha-tubulin was noticed to be a fixed point in this movement.

Methods: We performed comparative structural analysis of BtubA/B and $\alpha$ - and $\beta$-tubulin proteins using USCF Chimera10 and MEGA $X$ software. This data was obtained due to a comparison of 3 structures of microtubules with different nucleotides [pdb6DPU, 6DPV, 6DPW] and two structures for bacterial tubulins (miniMT [pdb5o09] and BtubA/B-dimer [pdb2BTQ]).

Results: We noticed that bacterial tubulins form shorter protofilaments in miniMT than eukaryotic ones. It can be explained as compaction in two sites instead of one site in eukaryotic MT. Also, the most motionless point of min MT turned out the same "anchor point." Phylogenetic analysis showed that this 
structure is very conservative in these orthologs. Moreover, the final state of both tubulins (GDP) repeats each other.

Conclusion: Our results suggest that bacterial tubulin can have movements through GTP hydrolysis similar to eukaryotic one. And it means that despite different amino acid sequences, bacterial and eukaryotic tubulins have similar keys structures for dynamic instability.

Key Words: $\alpha$-tubulin $\bullet \beta$-tubulin $・$ Prostecobacter $\bullet$ structure

This work was supported by the Russian Foundation for Basic Research (Grant No. 20-34-70159)

*Corresponding author: Liubov Makarova. E-mail: makarova.lo@phystech.edu

International Journal of Biomedicine. 2021;11 Suppl 1: S27-28.

doi: 10.21103/IJBM.11.Suppl_1.P36

(C)2021 International Medical Research and Development Corporation 